\title{
Biodiversidad en Nicaragua: contexto y estado actual
}

\author{
Antonio Mijail Pérez \\ Investigador de la Asociación Gaia
}

\section{Introducción}

De manera sintética se puede decir que la biodiversidad es el conjunto de los seres vivos existentes en el planeta. Históricamente este concepto abarcaba todas las formas de vida excepto el hombre, no obstante, numerosos autores cada vez más consideran al hombre como una parte inextricable del concepto de biodiversidad. De tal suerte surge una pregunta, isi la biodiversidad es tan importante, que incluye hasta nuestra propia especie, por qué tenemos tantos problemas para su conservación?

La antropización de diferentes tipos se traduce en deforestación, urbanización, erosión de los suelos, reforestación mono-específica que no restaura la biodiversidad del bosque primario, pérdida de hábitat para muchas especies, explosión descontrolada del turismo, globalización sin la participación de toda la sociedad, contaminación del aire, manejo inadecuado de los deshechos y consumo desmedido, que son amenazas constantes para preservar la biodiversidad del planeta ${ }^{1}$.

Una delas problemáticas que tienen los ciudadanos comunes preocupados por labiodiversidad y/o los estudiosos de la misma, es su desconocimiento. En el Cuadro 1 se presenta una aproximación a los datos sobre cantidades de especies por reinos, según Bellés², que nos permite visualizar lo que sabemos y lo que nos falta saber a nivel del planeta.

Cuadro 1.Datos generales de especies para el mundo

\begin{tabular}{|l|c|c|}
\hline Reinos & Especies descritas & Total de especies estimadas \\
\hline Bacterias & 4,000 & $1,000,000$ \\
\hline $\begin{array}{l}\text { Protoctistas (algas, } \\
\text { protozoos, etc.) }\end{array}$ & 80,000 & 600,000 \\
\hline Animales & $1,320,000$ & $10,600,000$ \\
\hline Hongos & 70,000 & $1,500,000$ \\
\hline Plantas & 270,000 & 300,000 \\
\hline Total & $\mathbf{1 , 7 4 4 , 0 0 0}$ & $\mathbf{c a .} \mathbf{1 4 , 0 0 0 , 0 0 0}$ \\
\hline
\end{tabular}

1 CRUZ, J. \& PÉREZ, A. M. (1990). Fauna amenazada de vertebrados de Cuba / Endangered Fauna of Cuban Vertebrates. La Habana: Academia de Ciencias de Cuba.

2 BELLÉS, X. (1998). Supervivientes de la biodiversidad. Barcelona: RubeSciencia. 


\section{Contexto}

Uno de los mayores problemas que tiene Nicaragua para conocer su biodiversidad es la disparidad de conocimiento que tenemos. Hay especies y grupos de especies de las que tenemos conocimientos muy avanzados y hay otras de las que no sabemos prácticamente nada.

En Nicaragua no hay una Academia de Ciencias ni un Instituto de Biodiversidad. Hay centros de investigación, ONG e investigadores dispersos que hacen estudios sobre biodiversidad, pero son pocos. Estudiando animales invertebrados - un mundo que incluye cientos de miles de especies- solamente habemos tres grupos de investigadores: nosotros en la Asociación Gaia, el Centro de Malacología y Diversidad Animal de la UCA -que trabaja con moluscos y otros invertebrados no insectos- y el Museo Entomológico de León, donde se trabaja con insectos. Somos los únicos equipos de investigadores trabajando en estos grupos de fauna. Hay otros que trabajan en temas aplicados, como el CIDEA de la UCA o el CIRA de la UNAN de Managua, pero en investigación en el ámbito de la sistemática, sólo los tres grupos mencionados. Trabajando con colecciones están, además, los dos Herbarios: el de la UCA y el de la UNAN de León. Los grupos que hacen algún tipo de trabajo con animales vertebrados no trabajan con colecciones, es decir, no hacen una colecta ni tienen material de respaldo, no tienen colecciones, ni alguna especie de museo.

De vertebrados no hay colecciones en Nicaragua. Lo más relevante es la colección de aves y reptiles que en su tiempo hizo el Padre Astorqui s.j. Está en un museo pequeño, que debido a las limitaciones presupuestarias de la UCA no podría crecer mucho más. Tiene el valor, hasta donde conozco, de ser el único museo que hay en Nicaragua de nuestros animales vertebrados. Hay también algunas colecciones de reptiles y anfibios guardados en alcohol que hizo el importante biólogo nicaragüense Jaime Villa, que actualmente reside fuera del país. Además, hay colecciones fraccionarias de animales de nuestro país en museos de Europa y Estados Unidos.

Recientemente se formó en Nicaragua una Asociación Científica Nicaragüense, pero su propósito es de índole más general y apunta al abordaje de problemáticas relacionadas con la investigación científica en sentido amplio, al debate sobre la construcción de un pensamiento científico y su inserción en el contexto actual de la tecnología.

\section{Principales amenazas de la biodiversidad en Nicaragua}

En nuestro criterio, las amenazas principales de la biodiversidad, su conservación y su estudio se pueden clasificar en cuatro principales -aunque desde luego, existe una relación directa o indirecta entre las mismas.

\section{Políticas:}

- Falta de programas de investigación y monitoreo de la biodiversidad

- Falta de fondos

- Falta de investigadores

- Falta de un instituto que reúna a los expertos en el tema

- Falta de publicaciones especializadas para la divulgación de los resultados 
Biológicas:

- Presencia de un número importante de especies invasoras

- Alto número de especies amenazadas

- Una superficie del territorio nacional dedicada a la conservación (áreas protegidas) que todavía es insuficiente

Académicas:

No existe una maestría especializante para los profesionales egresados de las carreras de biología de las universidades nacionales que son la cantera en que deben formarse los expertos en el tema. Recientemente la Universidad Americana (UAM) ha anunciado una Maestría en Biodiversidad, Conservación y Políticas Ambientales que deberá llenar este vacío. Sin embargo, el costo es elevado para los estándares nacionales y los organismos de cooperación en Nicaragua sólo financian a jóvenes que quieran realizar estudios fuera del país, con las consecuencias que esto supone para los programas de postgrado que se proponen dentro de Nicaragua.

Operativas:

La capacidad de inserción en el mundo laboral de los jóvenes egresados de las carreras afines a este campo o de los programas de postgrado existentes fuera del país es totalmente limitada.

\section{Esfuerzos para la conservación de la biodiversidad}

Existen esfuerzos de diferente índole para la conservación de la biodiversidad. El más clásico, podría decirse, es la implementación de áreas protegidas, aunque más recientemente los científicos de la conservación estamos evolucionando hacia el intento de implementación de mecanismos de conservación en sistemas productivos como los agroforestales y silvopastoriles.

\section{1. Áreas protegidas}

Según la UICN³ un área protegida se entiende como "un área de tierra y/o de mar dedicada especialmente a la protección y mantenimiento de la diversidad biológica, y de recursos naturales y culturales asociados, manejados mediante medios legales u otros que sean efectivos". De acuerdo con el Reglamento de áreas protegidas de Nicaragua y la Ley 217, Ley General del Medio Ambiente, son áreas protegidas "las que tienen por objeto la conservación, el manejo racional y la restauración de la flora, fauna silvestre y otras formas de vida, así como la biodiversidad y la biósfera, se pretende con ello restaurar y conservar fenómenos geomorfológicos, sitios de importancia histórica, arqueológica, cultural, escénicos o recreativos"4.

3 IUCN (1994). Guidelines for Protected Area Management Categories. Gland, Switzerland and Cambridge, UK: IUCN.

4 Ley 217. Ley General del Medio Ambiente y los Recursos Naturales y sus Reglamentos. Publicada en la Gaceta, Diario Oficial, No. 105, del 06 de junio de 1996. 
Las categorías de manejo nacionales y de la UICN se presentan en el Cuadro 2 y la Ilustración 1. Se incluye también un análisis que permite destacar las diferencias existentes entre estas categorías de cara a su mejor y más correcta aplicación. Al margen de los criterios utilizados para la selección de nuestras áreas protegidas, las mismas deben contener un importante porcentaje de la biodiversidad nacional, y actualmente las áreas protegidas representan el $17 \%$ del territorio nacional, lo que constituye un área relativamente significativa del mismo, aunque todavía insuficiente si se tiene en cuenta la presión que existe sobre las mismas. Un aspecto muy importante a destacar es la cantidad de áreas protegidas que existen en el país cuya categoría de manejo permite intervención humana (60 áreas).

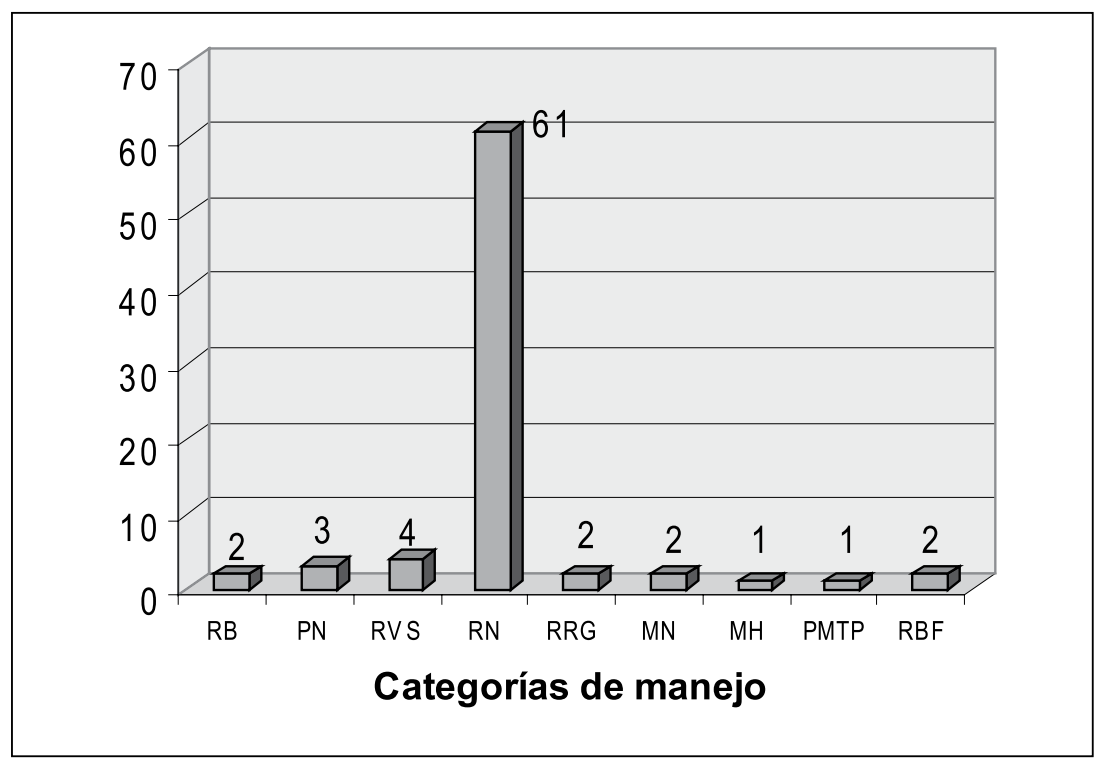

Ilustración 1. Áreas protegidas de Nicaragua por categoría

Cuadro 2. Categorías de manejo de áreas protegidas en Nicaragua

\begin{tabular}{|c|c|c|c|c|}
\hline $\begin{array}{c}\text { Categoría en } \\
\text { Nicaragua }\end{array}$ & $\begin{array}{c}\text { Categoría } \\
\text { UICN }\end{array}$ & Elemento diferencial & $\begin{array}{c}\text { Cantidad } \\
\text { de áreas }\end{array}$ & $\begin{array}{c}\text { Permite in- } \\
\text { tervención }\end{array}$ \\
\hline $\begin{array}{c}\text { Reserva } \\
\text { biológica }\end{array}$ & $\begin{array}{c}\text { Reserva natu- } \\
\text { ral estricta }\end{array}$ & $\begin{array}{l}\text { Áreas que poseen ecorregiones } \\
\text { y/o especies representativas. }\end{array}$ & $\mathbf{2}$ & No \\
\hline $\begin{array}{c}\text { Parque } \\
\text { nacional }\end{array}$ & $\begin{array}{c}\text { Parque } \\
\text { nacional }\end{array}$ & $\begin{array}{l}\text { Área idónea para proteger uno } \\
\text { o más ecosistemas y hábitats } \\
\text { así como rasgos de interés cul- } \\
\text { tural. }\end{array}$ & $\mathbf{3}$ & No \\
\hline
\end{tabular}




\begin{tabular}{|c|c|c|c|c|}
\hline $\begin{array}{c}\text { Refugio de } \\
\text { vida silvestre }\end{array}$ & $\begin{array}{l}\text { Área de mane- } \\
\text { jo de hábitats / } \\
\text { especies }\end{array}$ & $\begin{array}{l}\text { Área sujeta a intervención ac- } \\
\text { tiva para garantizar el manten- } \\
\text { imiento de los hábitats y/o para } \\
\text { satisfacer las necesidades de } \\
\text { determinadas especies o comu- } \\
\text { nidades animales. }\end{array}$ & 4 & $\mathbf{S i}$ \\
\hline $\begin{array}{l}\text { Reserva natu- } \\
\text { ral }\end{array}$ & $\begin{array}{l}\text { Área de mane- } \\
\text { jo de hábitats / } \\
\text { especies }\end{array}$ & $\begin{array}{l}\text { Área con especies de interés que } \\
\text { genere beneficios ambientales } \\
\text { de interés nacional o regional. }\end{array}$ & 60 & $\mathbf{S i}$ \\
\hline $\begin{array}{l}\text { Reserva de } \\
\text { recursos } \\
\text { genéticos }\end{array}$ & $\begin{array}{l}\text { Área de mane- } \\
\text { jo de hábitats / } \\
\text { especies }\end{array}$ & $\begin{array}{l}\text { Área que protege algunas espe- } \\
\text { cies por el interés de sus recur- } \\
\text { sos genéticos, los cuales pueden } \\
\text { ser utilizados para mejoramien- } \\
\text { to genético de especies de in- } \\
\text { terés económico o alimenticio. }\end{array}$ & 2 & $\begin{array}{c}\text { Sólo para } \\
\text { fines de } \\
\text { manejo }\end{array}$ \\
\hline $\begin{array}{l}\text { Monumento } \\
\text { nacional }\end{array}$ & $\begin{array}{l}\text { Monumento } \\
\text { natural }\end{array}$ & $\begin{array}{l}\text { Área que contiene rasgos na- } \\
\text { turales y/o histórico-culturales } \\
\text { de valor destacado o excepcio- } \\
\text { nal por su rareza implícita, sus } \\
\text { calidades representativas o es- } \\
\text { téticas. }\end{array}$ & 2 & $\mathbf{S i}$ \\
\hline $\begin{array}{l}\text { Monumento } \\
\text { histórico }\end{array}$ & $\begin{array}{l}\text { Monumento } \\
\text { natural }\end{array}$ & $\begin{array}{l}\text { Territorios que contienen uno o } \\
\text { varios rasgos culturales, históri- } \\
\text { cos o arqueológicos de impor- } \\
\text { tancia nacional o internacional } \\
\text { asociados a áreas naturales. }\end{array}$ & 1 & No \\
\hline $\begin{array}{l}\text { Paisaje } \\
\text { terrestre y } \\
\text { marino } \\
\text { protegido }\end{array}$ & $\begin{array}{l}\text { Paisaje } \\
\text { terrestre y } \\
\text { marino } \\
\text { protegido }\end{array}$ & $\begin{array}{l}\text { La interacción entre el hombre } \\
\text { y la naturaleza ha producido } \\
\text { una zona con importantes va- } \\
\text { lores estéticos, ecológicos y cul- } \\
\text { turales. }\end{array}$ & 1 & Si \\
\hline $\begin{array}{c}\text { Reserva de } \\
\text { biosfera }\end{array}$ & No tiene & $\begin{array}{l}\text { Áreas que integran diferentes } \\
\text { categorías de manejo y que ad- } \\
\text { ministradas integralmente lo- } \\
\text { gran un desarrollo sostenible. }\end{array}$ & 2 & $\mathbf{S i}$ \\
\hline
\end{tabular}

\subsection{Conservación fuera de las áreas protegidas}

Uno de los escenarios más importantes para la conservación de la biodiversidad fuera de áreas protegidas son los sistemas silvo-pastoriles, que son una modalidad de los sistemas agroforestales donde se desarrollan árboles y pasturas manejados en forma conjunta, 
cuyo objetivo es incrementar la productividad en forma sostenible, supliendo además otros beneficios ${ }^{5}$.

En el proyecto desarrollado por mi equipo de investigación ${ }^{6}$ se identificaron en 41 fincas ganaderas 212 especies de aves, lo que constituye el 30\% del total de especies del país (700), 56 especies de moluscos, para un 22\% del total nacional (257 especies) y 170 especies de árboles que constituyen el $2.6 \%$ de las plantas de Nicaragua.

Es evidente que la biodiversidad que puede ser conservada en sistemas productivos puede ser muy alta, lo que es notablemente interesante si se tiene en cuenta que un $37.49 \%$ del territorio nacional $\left(48,875.00 \mathrm{~km}^{2}\right.$ de $\operatorname{los} 130,373.47 \mathrm{~km}^{2}$ de área terrestre del país) corresponde a tierras de vocación agropecuaria ${ }^{7}$.

Aunque el citado proyecto duró solamente cinco años, muchos logros se consiguieron en el marco del mismo. Se produjo un aumento significativo de las cercas vivas en la zona, se produjo un aumento de árboles en potreros sin precedentes en otras zonas del país. Esta estrategia había demostrado ser de gran importancia para la conservación del suelo y el bienestar del ganado pero no había podido ser implementada hasta muy recientemente con la introducción de los pastos mejorados que crecen bajo sombra.
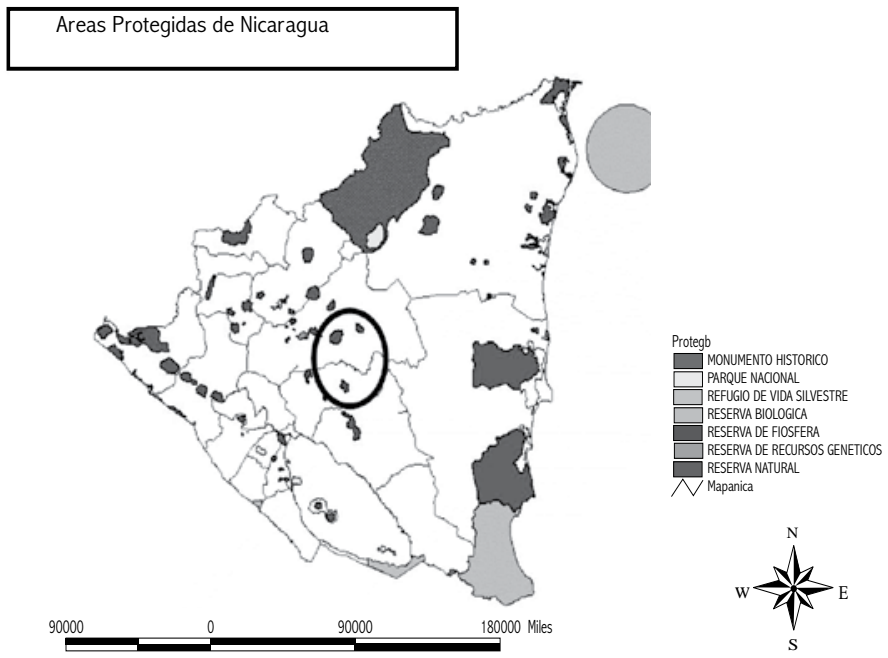

Ilustración 2. El área de estudio ubicada dentro del triángulo compuesto por las Reservas naturales Sierra Quirragua, Cerro Musún y Fila Masigüe al sur. Mapa tomado de CBMMARENA $^{8}$.

5 RADULOVICH, R. (1994) “Tecnologías productivas para sistemas agrosilvopecuarios de ladera con sequía estacional”. Serie Técnica, Informe Técnico No. 222, Turrialba, Costa Rica: Centro Agronómico Tropical de Investigación y Enseñanza (CATIE).

6 PÉREZ, A. M.; SOTELO, M.; RAMÍREZ, F.; RAMÍREZ, I.; LÓPEZ, A. \& SIRIA, I. (2006). "Conservación de la biodiversidad en sistemas silvopastoriles de Matiguás y Rio Blanco, Dpto de Matagalpa, Nicaragua”. Ecosistemas, 2006/3.

7 MARENA (2004). Indicadores ambientales de Nicaragua. Volumen I. Managua: Impresión comercial La Prensa.

8 CBM-MARENA (2001). Información CBM Nicaragua. MARENA-SICA/CCAD-PNUD/GEF-GTZ-PNUMA-BANCO MUNDIAL. Managua. En soporte CD. 


\subsection{Corredores biológicos}

Según CBM-MARENA, el Corredor Biológico Mesoamericano es una iniciativa política de los siete países centroamericanos -a la que se ha agregado México con sus estados del SurSureste- que persigue unificar y optimizar los esfuerzos regionales para la conservación y uso sostenible de la biodiversidad en beneficio de las generaciones actuales y futuras. Aunque el Corredor Biológico Mesoamericano como institución ya no existe, su planteamiento conceptual ha sido adoptado por muchos centroamericanos y aún hay acciones que buscan mecanismos de implementación desde los ministerios de los países de la región e incluso desde la CCAD. Algunos de los productos clave que ha legado el CBM a la región han sido los Programas PROMEBIO (Programa de Monitoreo de Biodiversidad), PERCON (Programa Estratégico Regional de Conectividad) y PERTAP (Programa Estratégico Regional de Trabajo en Áreas Protegidas), así como los indicadores regionales de biodiversidad.

\section{Marco legal de la conservación}

Uno de los aspectos más importantes para la conservación de la biodiversidad es la existencia de un marco legal. El marco nacional está conformado por políticas y leyes nacionales, de las cuales las más relevantes son:

Política ambiental y plan de acción (PNIC 2001-2005): Los Recursos Naturales y la Biodiversidad son patrimonio común de la sociedad y por tanto el Estado y todos los habitantes tienen el derecho y el deber de asegurar su uso sostenible, su accesibilidad y su calidad. Se considera el ambiente como la riqueza más importante del país por ser el determinante crítico de la cantidad, calidad y sustentabilidad de las actividades humanas y de la vida en general. El uso sostenible de los Recursos Naturales y la Biodiversidad contribuye a mejorar la calidad de vida reduciendo la brecha de pobreza y la vulnerabilidad ambiental. Las políticas y principios de equidad social y de género enmarcan la gestión ambiental o el criterio de prevención prevalece sobre cualquier otro en la gestión ambiental.

Política forestal: Sus principios son los mismos que aquellos de la política ambiental.

Política recursos hídricos: El agua es recurso natural finito y vulnerable, con valor económico, social y ambiental. Es patrimonio nacional denominado público, indispensable para satisfacer las necesidades básicas de la población. Cuenca es unidad de gestión territorial para administración y manejo integrado de los recursos hídricos. El desarrollo y la gestión del agua se basan en un enfoque participativo, involucrando a los usuarios, planificadores y tomadores de decisión a todos los niveles.

\section{Leyes nacionales:}

- Ley Orgánica del Instituto de Aguas y Alcantarillados (Ley 275) promulgada en 1979. Reforma la ley orgánica del Instituto Nicaragüense de Acueductos y Alcantarillados (INAA); tiene como objetivos regular, fiscalizar, y normar a nivel nacional el sector agua potable y alcantarillado sanitario; velar por los derechos de los consumidores y usuarios del servicio de alcantarillado sanitario.

- La ley de protección de suelos y control de erosión (S/N) promulgada en 1983. Tiene como 
objetivo proteger, preservar y controlar el efecto de la erosión en los suelos del territorio nacional, a fin de favorecer a la protección de cuencas hidrográficas y el desarrollo sostenible acorde con el medio ambiente.

- Ley de Municipios (Ley 40) promulgada en 1988. El papel del municipio, con respecto a la normación y control de los recursos naturales que se encuentran en su circunscripción, es muy reducido ${ }^{9}$. Una gran cantidad de cargas adicionales se establecen para el Municipio, pero ningún nivel decisorio.

- Ley General del Medio Ambiente (Ley 217) promulgada en 1996. Establece la evaluación de impacto ambiental, áreas protegidas, normas y procedimientos para la exportación, importación y reexportación de flora y fauna.

- Ley de conservación, fomento y desarrollo sostenible del sector forestal (Ley 462) promulgada en 2003. Tiene como objetivo establecer el marco legal para la conservación, fomento y desarrollo sostenible del sector forestal tomando como base fundamentalmente el manejo forestal del bosque natural, el fomento de las plantaciones, la protección, conservación y restauración de áreas forestales.

- Reglamento Ley 462.

- Ley de veda para el corte, aprovechamiento y comercialización del recurso forestal (Ley 585) promulgada en 2006.

Como puede observarse existe un marco legal abundante y bien construido, pero en muchas ocasiones no se implementa por limitaciones de diferentes tipos.

\section{Retos futuros}

\section{Políticos}

Existen algunos retos que se podrían clasificar como de corto plazo. El primero debería ser la publicación de la Ley de biodiversidad que fue elaborada desde hace más de dos años por un grupo de expertos de una comisión interinstitucional. Se debería impulsar también la consulta de la Política Nacional de Biodiversidad que está elaborada hace más de un año.

\section{Operativos}

Para orientar las acciones de conservación dela biodiversidad, el primer paso es la elaboración de una línea de base de país. Este estudio permitiría homogeneizar el conocimiento de los grupos más emblemáticos de la biodiversidad por áreas del país y ha sido ya realizado por todos los países de la región excepto Nicaragua.

Un siguiente paso también perentorio es la elaboración de una lista actualizada de las especies amenazadas o en peligro de extinción, de tal suerte es loable el trabajo titulado "Listas de fauna de importancia para la conservación en Centroamérica y México" ${ }^{10}$, en el que se reúnen a rasgos generales los taxa del área que caen en alguna de las categorías antes citadas.

9 Comunicación personal del Dr. Alejandro Bravo, ex Director Ejecutivo de AMUNIC y profesor horario de la UCA.

10 CCAD (1999). Lista de fauna de importancia para la conservación en Centroamérica y México: listas rojas, listas oficiales y especies en apéndices CITES. WWF, UICN, SICA. 


\section{Filosóficos}

Se debe hacer un esfuerzo por concentrar los esfuerzos de los organismos de la cooperación, de la empresa privada y de la banca nacional en el apoyo de las iniciativas que aborden los grandes temas actuales como la conservación de la biodiversidad y el cambio climático, la generación de energías alternativas y el desarrollo de sistemas productivos ecoamigables, entre otros aspectos.

Se podría decir además y como colofón, que es vital contar con el apoyo de todos los sectores para la formación de un pensamiento de conservación en la sociedad nicaragüense que permita revertir el acelerado proceso de deforestación y contaminación que están experimentando actualmente nuestros ecosistemas. 